Military Technical College Kobry El-Kobbah, Cairo, Egypt

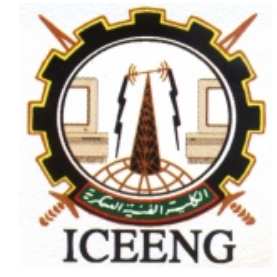

\author{
$8^{\text {th }}$ International Conference \\ on Electrical Engineering \\ ICEENG 2012
}

\title{
Effective of hall current on unsteady Couette flow with heat transfer in the presence of a heat source and uniform suction and injection
}

\author{
By \\ Hazem Ali Attia * Karem Mahmoud Ewis ** Ibrahim Hamdy Abd Elmaksoud***
}

\begin{abstract}
:
The unsteady magnetohydrodynamic (MHD) Couette flow with heat transfer of an electrically conducting, viscous, incompressible fluid bounded by two parallel insulating porous plates is studied in the presence of uniform suction and injection and a heat source considering the Hall effect. An externally applied uniform magnetic field as well as a uniform suction and injection are applied in the direction perpendicular to the plates. A uniform and constant pressure gradient is imposed in the axial direction. The two plates are kept at different but constant temperatures while the Joule and viscous dissipations are included in the energy equation. The effect of the Hall current and the uniform suction and injection on both the velocity and temperature distributions is investigated and interesting results are presented for different values of the parameters of the problem.
\end{abstract}

\section{Keywords:}

Couette flow, hydromagnetic, heat transfer, Hall current, numerical solution.

* Faculty of Engineering, Fayoum University, Fayoum, Egypt

** Faculty of Engineering, Fayoum University, Fayoum, Egypt

*** Faculty of Engineering, Fayoum University, Fayoum, Egypt 


\section{Introduction:}

The magnetohydrodynamic (MHD) flow between two parallel plates, known as Hartmann flow, is a classical problem that has many applications in MHD power generators, MHD pumps, accelerators, aerodynamic heating, electrostatic precipitation, polymer technology, petroleum industry, purification of crude oil and fluid droplets and sprays. Hartmann and Lazarus [1] studied the influence of a transverse uniform magnetic field on the flow of a conducting fluid between two infinite parallel, stationary, and insulated plates. Then, a lot of research work concerning the Hartmann flow has been obtained under different physical effects [2-10]. In most cases the Hall and ion slip terms were ignored in applying Ohm's law as they have no marked effect for small and moderate values of the magnetic field. However, the current trend for the application of MHD is towards a strong magnetic field, so that the influence of electromagnetic force is noticeable [5]. Under these conditions, the Hall current and ion slip are important and they have a marked effect on the magnitude and direction of the current density and consequently on the magnetic force term. Tani [7] studied the Hall effect on the steady motion of electrically conducting and viscous fluids in channels. Soudalgekar et al. [89] studied the effect of the Hall currents on the steady MHD Couette flow with heat transfer. The temperatures of the two plates were assumed either to be constant [8] or to vary linearly along the plates in the direction of the flow [9]. Abo-El-Dahab [10] studied the effect of Hall current on the steady Hartmann flow subjected to a uniform suction and injection at the bounding plates. Later, Attia [11] extended the problem to the unsteady state with heat transfer, with constant pressure gradient applied.

In the present paper, the unsteady Couette flow and heat transfer of an incompressible, viscous, electrically conducting fluid between two infinite insulating horizontal porous plates are studied with the consideration of the Hall current and in the presence of a heat source. The upper plate is moving with a uniform velocity while the lower plate remains stationary. The fluid is acted upon by a constant pressure gradient, a uniform suction and injection and a uniform magnetic field perpendicular to the plates. The induced magnetic field is neglected by assuming a very small magnetic Reynolds number $[4,5]$. The two plates are maintained at two different but constant temperatures. This configuration is a good approximation of some practical situations such as heat exchangers, flow meters, and pipes that connect system components. The cooling of these devices can be achieved by utilizing a porous surface through which a coolant, either a liquid or gas, is forced. Therefore, the results obtained here are important for the design of the wall and the cooling arrangements of these devices. A numerical solution for the governing equations including the Joule and viscous dissipations is developed. The effect of the magnetic field, the Hall current and the suction and injection on both the velocity and temperature distributions is reported. 


\section{Governing Equations:}

The two insulating plates are located at the $y= \pm h$ planes and extend from $x=-\infty$ to $\infty$ and $z=-\infty$ to $\infty$. The upper plate is moving with a uniform velocity $U_{\mathrm{o}}$ while the lower plate remains fixed. The lower and upper plates are kept at the two constant temperatures $T_{1}$ and $T_{2}$, respectively, where $T_{2}>T_{1}$ and a heat source is included. The fluid flows between the two plates under the effect of a constant pressure gradient $d P / d x$ in the axial $x$-direction, and a uniform suction from above and injection from below which are applied at $t=0$ with velocity $v_{o}$. The whole system is subjected to a uniform magnetic field $B_{\mathrm{o}}$ in the positive $y$-direction. This is the total magnetic field acting on the fluid since the induced magnetic field is neglected. From the geometry of the problem, it is evident that all quantities are independent of $x$ and $z$-coordinates apart from the pressure gradient $d P / d x$. The existence of the Hall term results in a $z$-component of the velocity. Thus, the velocity vector of the fluid is

$v(y, t)=u(y, t) i+v_{o} j+w(y, t) k$

The initial and boundary conditions are: $u=w=0$ at $t \unlhd), u=w=0$ at $y=-h$ for $t>0$ and $u=U_{\mathrm{o}}$ and $w=0$ at $y=h$ for $t>0$. The temperature $T(y, t)$ at any point in the fluid satisfies both the initial and boundary conditions $T=T_{1}$ at $\left.t \unlhd\right), T=T_{2}$ at $y=+h$, and $T=T_{1}$ at $y=-h$ for $t>0$. The fluid flow is governed by the momentum equation

$\rho \frac{D v}{D t}=\mu \nabla^{2} v-\nabla P+J \wedge B_{o}$

If the Hall term is retained, the current density $\boldsymbol{J}$ is given by

$$
J=\sigma\left(v \wedge B_{o}-\beta\left(J \wedge B_{o}\right)\right)
$$

This equation may be solved in $\boldsymbol{J}$ yielding

$$
J \wedge B_{o}=-\frac{\sigma B_{o}^{2}}{1+m^{2}}((u+m w) i+(w-m u) k)
$$

where $m=\sigma \beta B_{o}$, is the Hall parameter [4]. Thus, in terms of Eq. (2), the two components of Eq. (1) read [12]

$$
\rho \frac{\partial u}{\partial t}+\rho v_{o} \frac{\partial u}{\partial y}=-\frac{d P}{d x}+\mu \frac{\partial^{2} u}{\partial y^{2}}-\frac{\sigma B_{o}^{2}}{1+m^{2}}(u+m w),
$$


$\rho \frac{\partial w}{\partial t}+\rho v_{o} \frac{\partial w}{\partial y}=\mu \frac{\partial^{2} w}{\partial y^{2}}-\frac{\sigma B_{o}^{2}}{1+m^{2}}(w-m u)$,

The temperature distribution is governed by the energy equation [12]

$$
\rho c_{p} \frac{\partial T}{\partial t}+\rho c_{p} v_{o} \frac{\partial T}{\partial y}=k \frac{\partial^{2} T}{\partial y^{2}}+Q T+\mu\left(\left(\frac{\partial u}{\partial y}\right)^{2}+\left(\frac{\partial w}{\partial y}\right)^{2}\right)+\frac{\sigma B_{o}^{2}}{1+m^{2}}\left(u^{2}+w^{2}\right),
$$

The second and third terms on the right side represent the viscous and Joule dissipations, respectively. Introducing the following non-dimensional quantities

$\hat{x}=\frac{x}{h}, \hat{y}=\frac{y}{h}, \hat{z}=\frac{z}{h}, \hat{u}=\frac{u}{U_{o}}, \hat{P}=\frac{P}{\rho U_{o}^{2}}, \hat{t}=\frac{t U_{o}}{h}$,

$\operatorname{Re}=\rho h U_{o} / \mu$, is the Reynolds number,

$S=v_{o} / U_{o}$, is the suction parameter,

$\operatorname{Pr}=\mu c_{p} / k$, is the Prandtl number,

$E c=U_{o}^{2} / c_{p}\left(T_{2}-T_{1}\right)$, is the Eckert number,

$H a^{2}=\sigma B_{o}^{2} h^{2} / \mu$, where Ha is the Hartmann number,

$\hat{Q}=Q U_{0} /\left(\rho h c_{p}\right)$ is the dimensionless heat generation coefficient

the basic Eqs. (3)-(5) are written as (the hats are dropped for convenience)

$\frac{\partial u}{\partial t}+S \frac{\partial u}{\partial y}=-\frac{d P}{d x}+\frac{1}{\operatorname{Re}} \frac{\partial^{2} u}{\partial y^{2}}-\frac{H a^{2}}{\operatorname{Re}\left(1+m^{2}\right)}(u+m w)$,

$\frac{\partial w}{\partial t}+S \frac{\partial w}{\partial y}=\frac{1}{\operatorname{Re}} \frac{\partial^{2} w}{\partial y^{2}}-\frac{H a^{2}}{\operatorname{Re}\left(1+m^{2}\right)}(w-m u)$,

$\frac{\partial T}{\partial t}+S \frac{\partial T}{\partial y}=\frac{1}{\operatorname{Re} \operatorname{Pr}} \frac{\partial^{2} T}{\partial y^{2}}+Q T+\frac{E c}{\operatorname{Re}}\left(\left(\frac{\partial u}{\partial y}\right)^{2}+\left(\frac{\partial w}{\partial y}\right)^{2}\right)+\frac{E c H a^{2}}{\operatorname{Re}\left(1+m^{2}\right)}\left(u^{2}+w^{2}\right)$,

The initial and boundary conditions for the velocity become

$t \leq 0: u=w=0, t>0: u=w=0, y=-1, u=1, w=0, y=1$, 
and the initial and boundary conditions for the temperature are given by

$t \leq 0: T=0, t>0: T=1, y=+1, T=0, y=-1$.

\section{Results and Discussion:}

Equations (6)-(8) are solved numerically using finite differences [13] under the initial and boundary conditions (9) and (10) to determine the velocity and temperature distributions for different values of the parameters $H a$ and $S$. The Crank-Nicolson implicit method is applied and the finite difference equations are written at the midpoint of the computational cell and the different terms are replaced by their secondorder central difference approximations in the $y$-direction. The diffusion term is replaced by the average of the central differences at two successive time levels. The viscous and Joule dissipation terms are evaluated using the velocity components and their derivatives in the $y$-direction which are obtained from the exact solution. Finally, the block tri-diagonal system is solved using Thomas' algorithm. All computations are carried out for $d P / d x=3, R e=1, P r=1$ and $E c=0.2$.

Figure 1 presents the profiles of the velocity components $u$ and $w$ and the temperature $T$ for different values of time $t$ and for $H a=1, m=3, S=1$ and $Q=0.1$. It is seen that the velocity components and temperature reaches the steady state monotonically with time. Also the velocity component $u$ reaches the steady state faster than $w$ which, in turn, reaches the steady state faster than $T$ because $u$ is the source of $w$, while both $u$ and $w$ act as sources for the temperature.

Figure 2 indicates that the time progression of $u$ and $w$ at the centre of the channel $y=0$ for different values of the Hall parameter $m$ and for $H a=1, S=0$ and $Q=0.1$. It is clear from Fig. 2a that increasing the parameter $m$ increases $u$ because the effective conductivity $\left(\sigma /\left(1+m^{2}\right)\right)$ decreases with increasing $m$ which reduces the magnetic resistive force on $u$. In Fig. $2 \mathrm{~b}$, the velocity component $w$ increases with increasing the parameter $m$ slightly ( $m=0$ to 1 ), since increasing $m$ increases the driving force term $\left(m H a^{2} u /\left(1+m^{2}\right)\right)$ in Eq. (7) which pumps the flow in the z-direction. However, increasing $m$ more decreases the effective conductivity that results in a reduced driving force and then, decreases $w$. It is clear from Fig. 2c that increasing $m$ decreases $T$ for all $t$ due to decreasing the effect of the Joule dissipation.

Figure 3 presents the time progression of $u, w$ and $T$ at the centre of the channel for different values of the Hartmann number $H a$ and for $m=3, S=0$ and $Q=0.1$. Figure 3a indicates that increasing $\mathrm{Ha}$ decreases $u$ as a result of increasing the damping force on $u$. Figure $3 \mathrm{~b}$ indicates that increasing $\mathrm{Ha}$ increases $w$ since it increases the driving force on $w$. Figure $3 \mathrm{c}$ depicts that for small $t$, increasing $\mathrm{Ha}$ increases $\mathrm{T}$ due to the increment in the Joule dissipation. But, for large $t$, increasing $H a$ decreases $T$ as a result 
of decreasing the velocities $u$ and $w$ and consequently decreases the viscous and Joule dissipations.

Figure 4 presents the time progression of $u, w$ and $T$ at the centre of the channel for different values of the suction parameter $S$ and for $H a=1, m=3$ and $Q=0.1$. Figures $4 \mathrm{a}$ and $4 \mathrm{~b}$ indicate that increasing the suction decreases both $u$ and $w$ due to the convection of the fluid from regions in the lower half to the centre which has higher fluid speed. Figure $4 \mathrm{c}$ shows that increasing $S$ decreases the temperature at the centre of the channel due to the influence of convection in pumping the fluid from the cold lower half towards the centre of the channel.

Figure 5 presents the time progression of $T$ at the centre of the channel for different values of the parameter $Q$ and for $H a=1, m=3$ and $S=0$. The figure indicates that increasing $Q$ increases the temperature at the centre of the channel and its steady state time. Table 1 presents the variation of the Nusselt number at the lower plate with time for various values of the parameters $S$ and $Q$ and for $H a=1$ and $m=3$. It is clear that increasing $Q$ increases the Nusselt number for all values of the suction parameter $S$ and for all time. On the other hand, increasing the suction parameter $S$ decreases the Nusselt number for all values of $Q$ and the time $t$. 

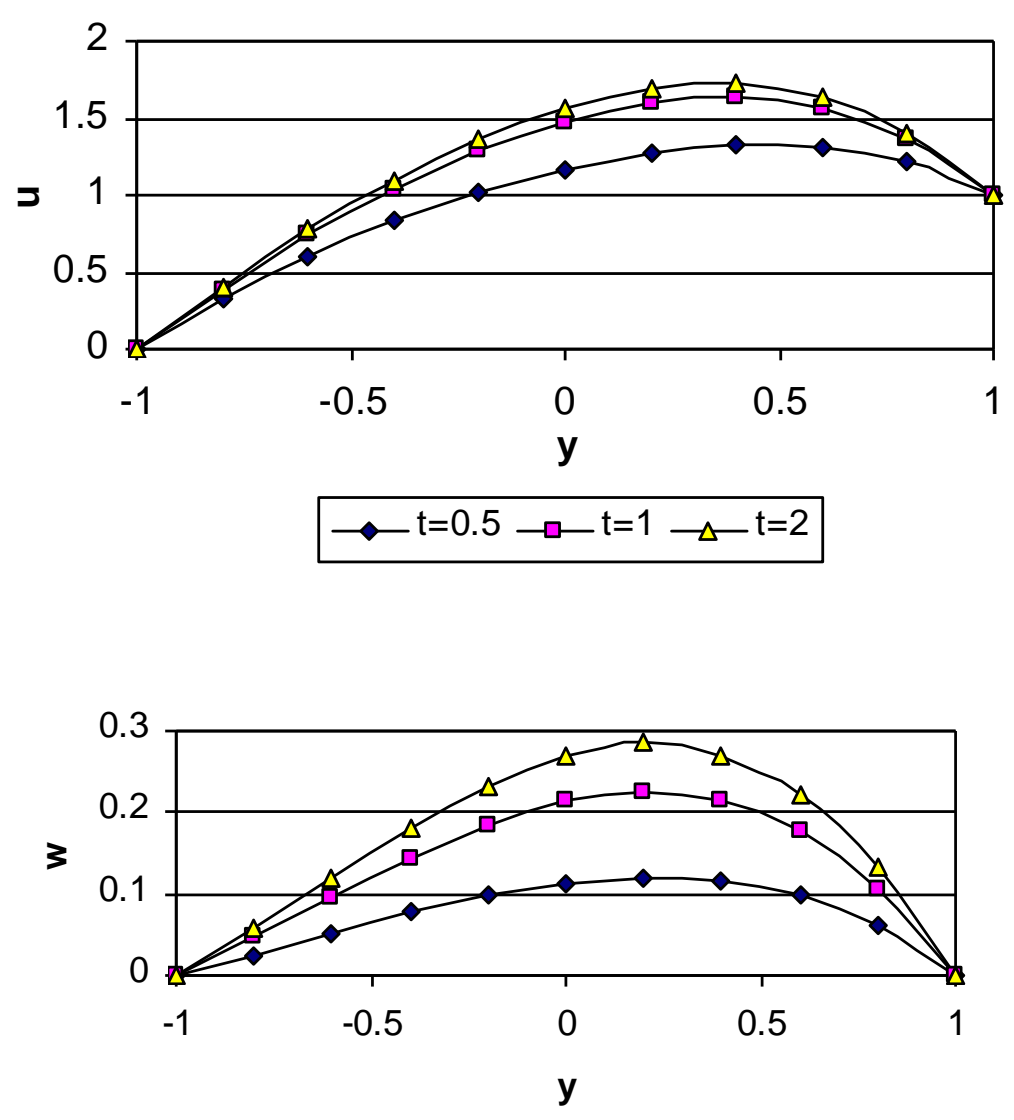

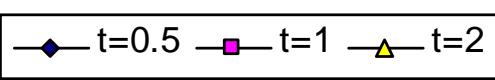

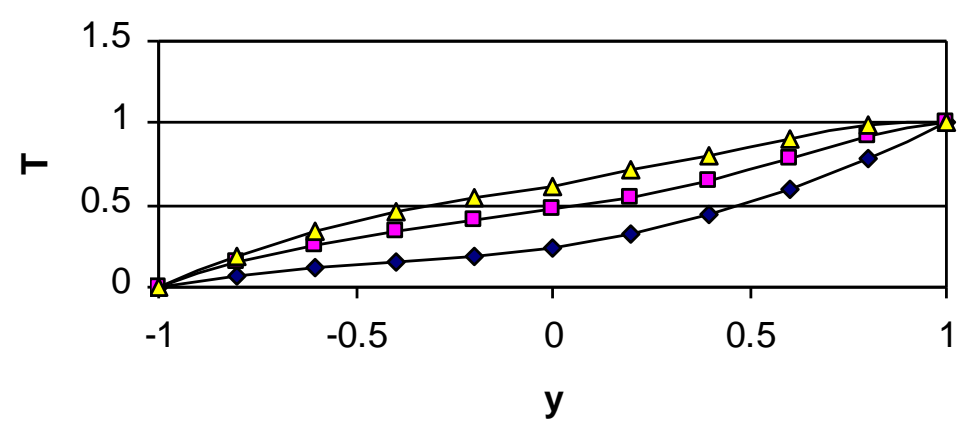

$\multimap \mathrm{t}=0.5 \multimap \square^{\mathrm{t}=1} \triangleleft \mathrm{t}^{\mathrm{t}=2}$

Figure (1): Time development of the profile of: (a) $u$; (b) $w$; and (c) $T(H a=1, m=3$, $S=1$ and $\mathrm{Q}=0.1$ ) 


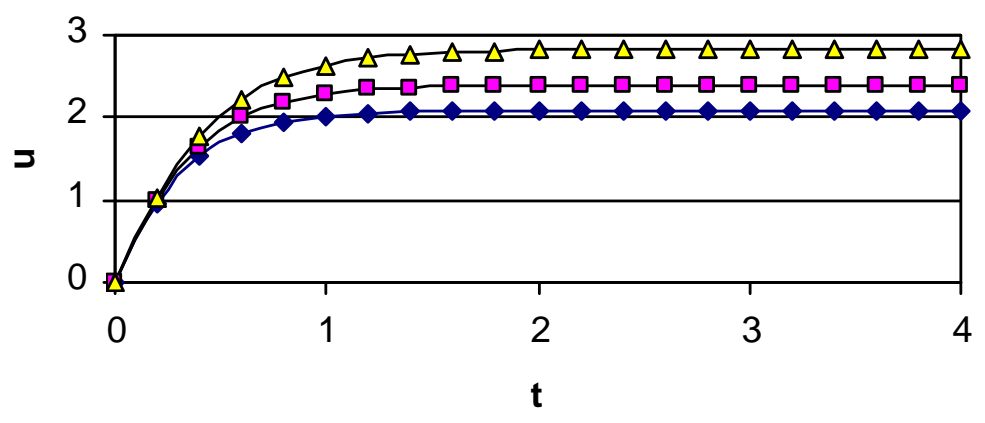

$\neg m=0 \multimap m=1 \multimap m=3$

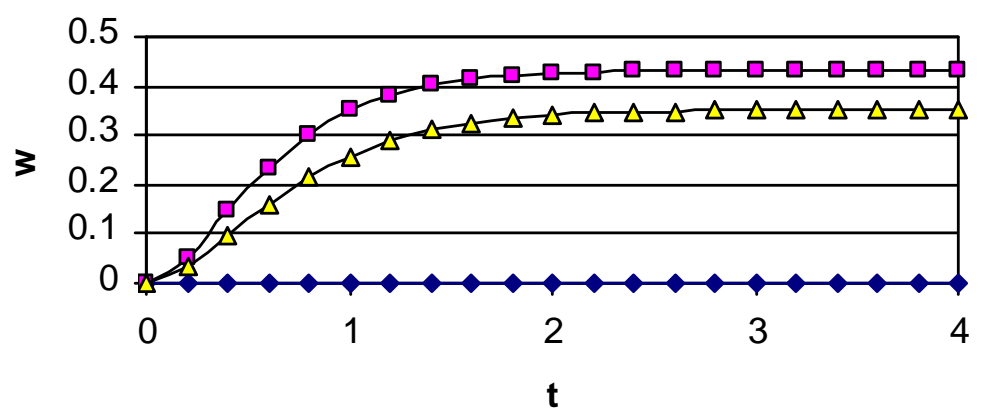

$\neg \mathrm{m}=0 \multimap \mathrm{m}=1 \multimap \Delta \mathrm{m}=3$

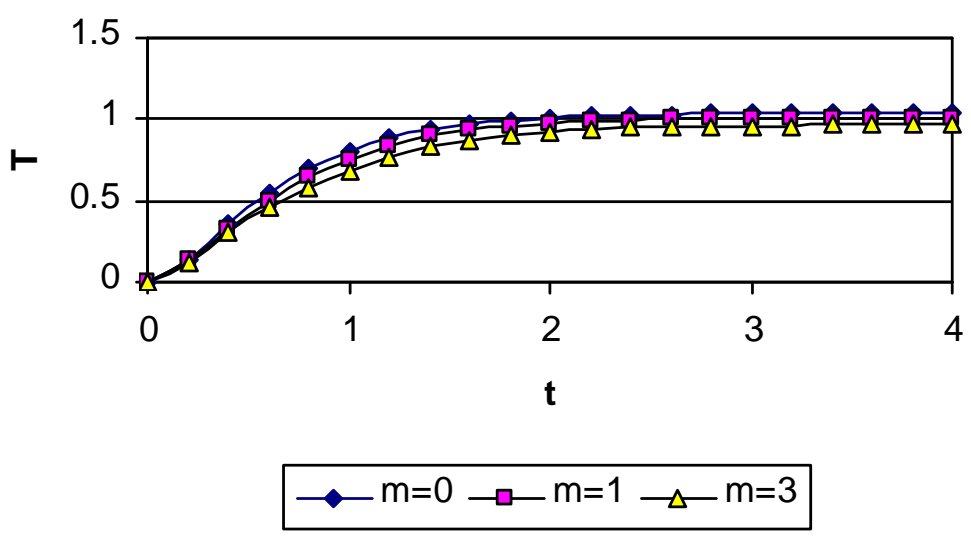

Figure (2): Effect of $m$ on the time variation of: (a) $u$ at $y=0$; (b) $w$ at $y=0$ and (c) $T$ at $y=0 .(H a=1$ and $S=0, \mathrm{Q}=0.1)$ 


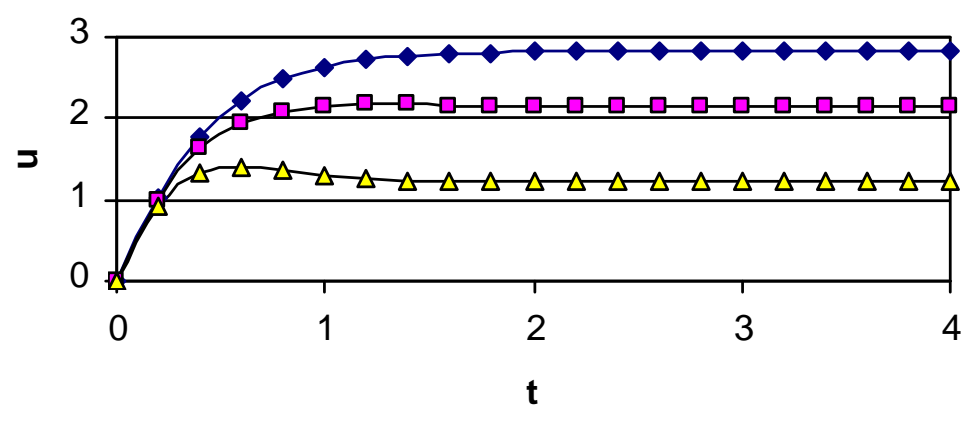

$\neg \mathrm{Ha}=1 \multimap-\mathrm{Ha}=2 \neg \mathrm{Ha}=3$

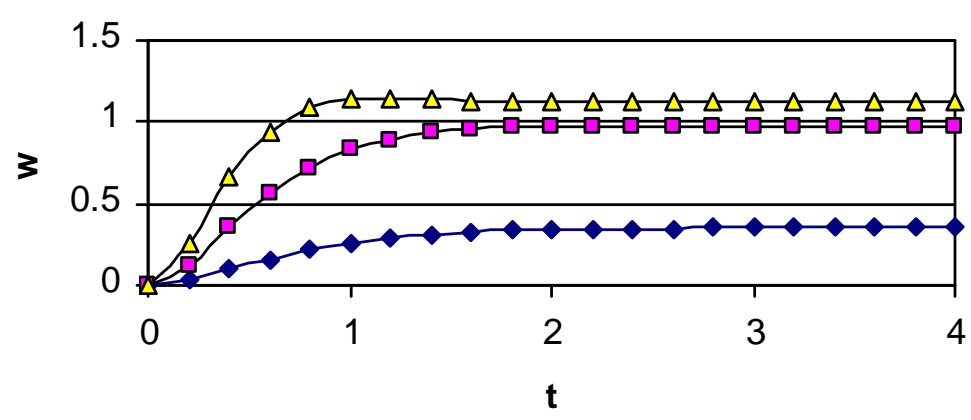

$\multimap-\mathrm{Ha}=1 \neg-\mathrm{Ha}=2 \neg-\mathrm{Ha}=3$

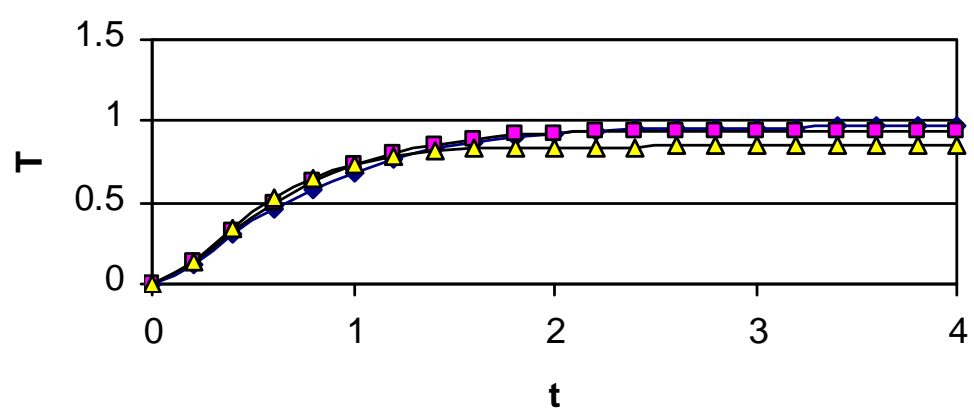

$\multimap \multimap \mathrm{Ha}=1 \rightarrow-\mathrm{Ha}=2 \multimap-\mathrm{Ha}=3$

Figure (3): Effect of $\mathrm{Ha}$ on the time variation of: (a) $u$ at $y=0$; (b) $w$ at $y=0$ and (c) $T$ at $y=0 .(m=3$ and $S=0, \mathrm{Q}=0.1)$ 

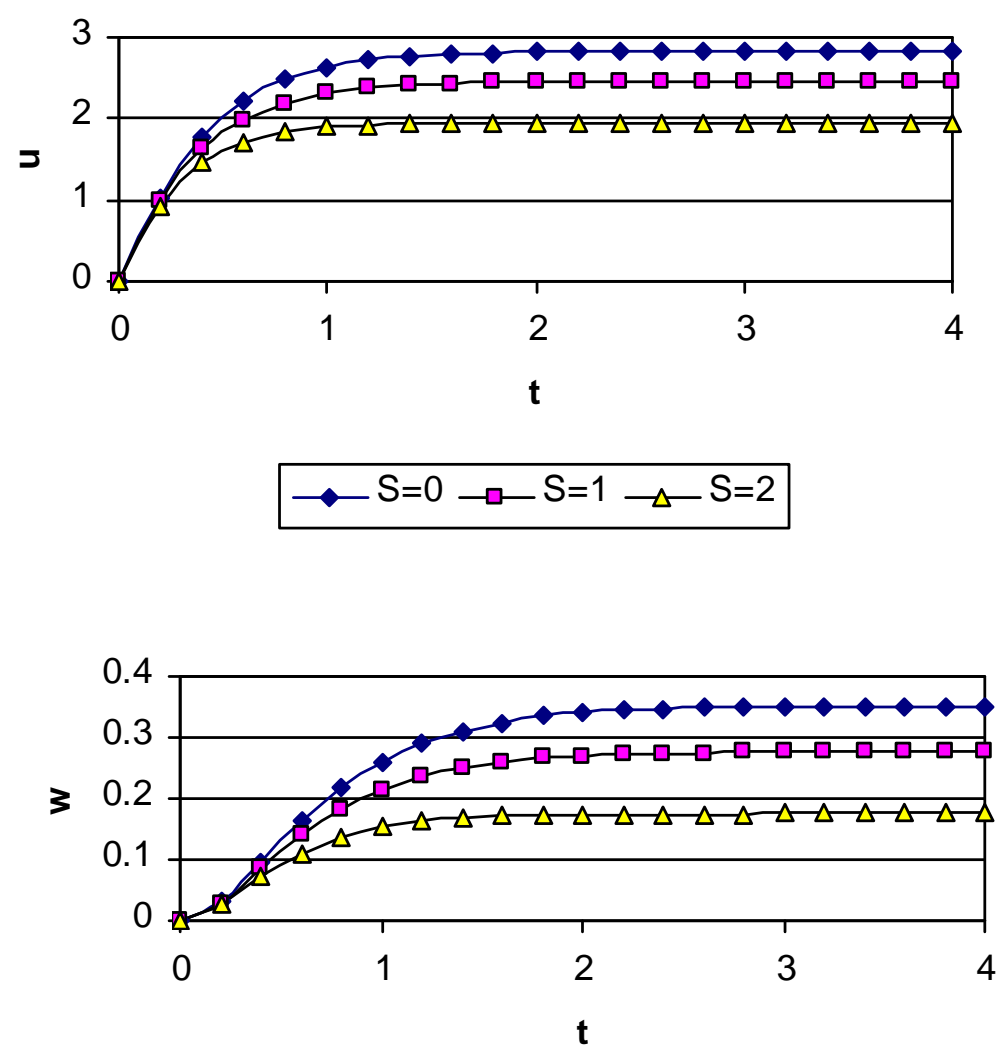

$\multimap \mathrm{S}=0 \multimap \mathrm{S}=1 \multimap \mathrm{S}=2$

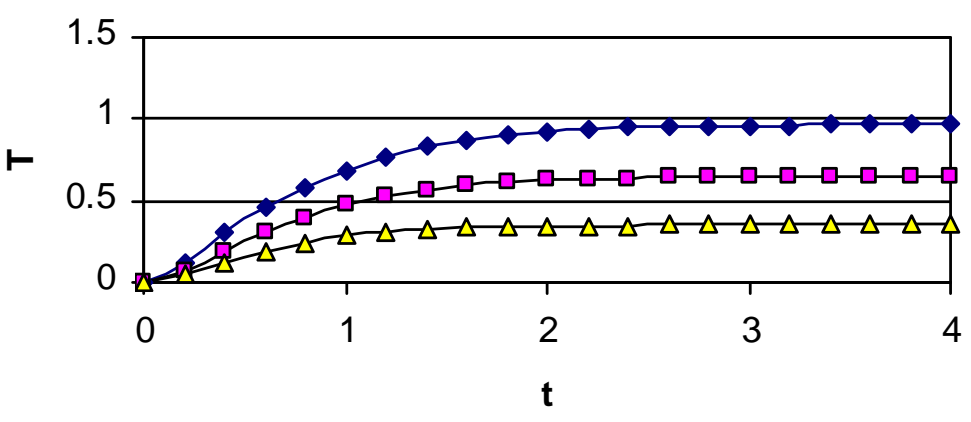

$\neg \mathrm{S}=0 \multimap \mathrm{S}=1 \multimap \mathrm{S}=2$

Figure (4): Effect of $m$ on the time variation of: (a) $u$ at $y=0$; (b) $w$ at $y=0$; and (c) $T$ at $y=0 .(H a=1$ and $m=3)$ 


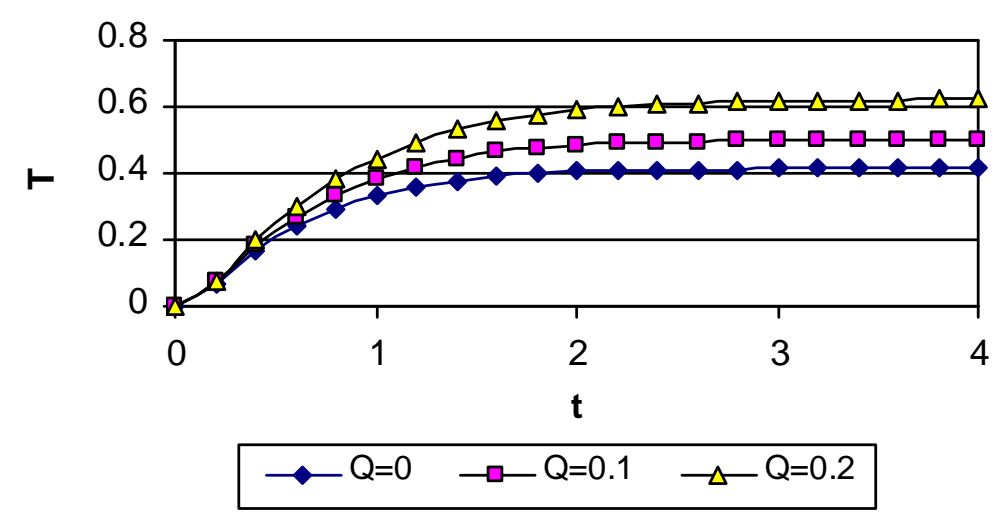

Figure (5): Effect of $Q$ on the time variation of: $T$ at $y=0 .(S=1, H a=1$ and $m=3)$

\section{Conclusions:}

The unsteady MHD Couette flow with heat transfer in an electrically conducting fluid under the influence of an applied uniform magnetic field has been studied considering the Hall effect in the presence of uniform suction and injection and an external heat source. Introducing the Hall term gives rise to a velocity component $w$ in the $z$-direction which affects the main velocity $u$ in the $x$-direction. The effect of the magnetic field, the Hall parameter and the suction and injection velocity on the velocity and temperature distributions are investigated. As time develops, increasing the Hall parameter $m$ increases the velocity component $u$. On the other hand, increasing $m$ increases the velocity component $w$ for small $m$ but decreases it for large $m$. It is also found, that the effect of both parameters $\mathrm{Ha}$ and $m$ on the temperature $T$ depends on time. 
Proceedings of the $\boldsymbol{8}^{\text {th }}$ ICEENG Conference, 29-31 May, 2012

\section{References:}

[1] J. Hartmann and F. Lazarus, Kgl. Danske Videnskab. Selskab, Mat.-Fys. Medd. Vol. 15, P. 6-7, 1937.

[2] I.N. Tao, Magnetohydrodynamic effects on the formation of Couette flow, J. of Aerospace Sci., Vol. 27, pp. 334, 1960.

[3] R.A. Alpher, Heat transfer in magnetohydrodynamic flow between parallel plates, Int. J. Heat and Mass Transfer, Vol. 3, pp. 108, 1961.

[4] G.W. Sutton and A. Sherman, Engineering Magnetohydrodynamics, McGrawHill Book Co. 1965.

[5] K. Cramer and S.-I. Pai, Magnetofluid dynamics for engineers and applied physicists, McGraw-Hill Book Co., 1973.

[6] S.D. Nigam and S.N. Singh, Heat transfer by laminar flow between parallel plates under the action of transverse magnetic field, Quart. J. Mech. Appl. Math., Vol. 13, pp. 85, 1960.

[7] I. Tani, Steady motion of conducting fluids in channels under transverse magnetic fields with consideration of Hall effect, J. of Aerospace Sci., Vol. 29, pp. 287, 1962.

[8] V.M. Soundalgekar, N.V. Vighnesam, and H.S. Takhar, Hall and Ion-slip effects in MHD Couette flow with heat transfer, IEEE Transactions on Plasma Science, Vol. PS-7, No. 3 (Sept.), pp. 178-182, 1979.

[9] V.M. Soundalgekar and A.G. Uplekar, Hall effects in MHD Couette flow with heat transfer, IEEE Transactions on Plasma Science, Vol. PS-14, No. 5 (Oct.), pp. 579-583, 1986.

[10] E.M.H. Abo-El-Dahab, Effect of Hall currents on some magnetohydrodynamic flow problems, Master Thesis, Dept. of Math., Fac. of Science, Helwan Univ., Egypt, 1993.

[11] H.A. Attia, Hall current effects on the velocity and temperature fields of an unsteady Hartmann flow, Can. J. Phys., Vol. 76, No. 9, pp. 739, 1998.

[12] H. Schlichting, Boundary layer theory, McGraw-Hill Book Co., 1968.

[13] W.F. Ames, Numerical solutions of partial differential equations. 2nd ed., Academic Press, New York, 1977. 


\section{Nomenclatures:}

$\rho \ldots$ the density of the fluid

$\sigma \ldots$ the fluid electrical conductivity

$\beta \ldots$ the Hall factor

... the viscosity of the fluid

$B_{o} \ldots$ the magnetic field

$c_{p} \ldots$ the specific heat capacity

Ec... the Eckert number

$H a \ldots$ the Hartmann number

$J \ldots$ the current density

$k \ldots$ the thermal conductivity of the fluid.

$m \ldots$ the Hall parameter

Pr... the Prandtl number

$R e \ldots$ the Reynolds number

$S \ldots$ the suction parameter

$t \ldots$ Time

$T \ldots$ the temperature

$T_{1} \ldots$ The lower constant temperature

$T_{2} \ldots$ The upper constant temperature

$u \ldots$ the fluid velocity in direction $x$

$w \ldots$ the fluid velocity in direction $z$ 